\title{
Efficacy and safety of anticoagulation in non-malignant portal vein thrombosis in patients with liver cirrhosis: a systematic review and meta-analysis
}

\author{
Sami Ghazaleha , Azizullah Beran ${ }^{a}$, Kanana Aburayyana ${ }^{a}$ Christian Nehme ${ }^{a}$, Dipen Patela, \\ Yasmin Khadera, Sachit Sharma ${ }^{a}$, Muhammad Aziza, Yousef Abdel-Aziz ${ }^{\mathrm{b}}$, Tariq Hammad', Ali Nawras ${ }^{\mathrm{d}}$ \\ University of Toledo, OH; University of Tennessee, Memphis, TN; Baylor College of Medicine, Houston, TX, USA
}

\section{Abstract}

\begin{abstract}
Background The role of anticoagulation in treating non-malignant portal vein (PV) thrombosis (PVT) in patients with liver cirrhosis remains unclear. In our meta-analysis, we aimed to evaluate the efficacy and safety of anticoagulation for the treatment of non-malignant PVT in these patients.
\end{abstract}

Methods We conducted a meta-analysis to estimate the effects of anticoagulation on nonmalignant PVT in patients with liver cirrhosis. We assessed the rates of PV recanalization, variceal bleeding, and any bleeding.

Results We included 9 observational studies which involved 474 patients. The rate of PV recanalization was significantly higher in patients who received anticoagulation compared to those who did not: $65.2 \%$ vs. $25.2 \%$; relative risk (RR) $2.31,95 \%$ confidence interval (CI) 1.80-2.96; $\mathrm{P}<0.00001$. Variceal bleeding was significantly lower in patients who received anticoagulation: $0.1 \%$ vs. $18.5 \%$; RR $0.15,95 \%$ CI $0.04-0.55 ; \mathrm{P}=0.004$. Any bleeding was similar between patients who received anticoagulation and those who did not: $10.3 \%$ vs. $22.7 \%$; RR $0.43,95 \%$ CI $0.09-1.99$; $\mathrm{P}=0.28$.

Conclusions Anticoagulation use increased the rate of $\mathrm{PV}$ recanalization in cirrhotic patients with non-malignant PVT. Anticoagulation decreased the rate of variceal bleeding and did not increase the rate of any bleeding.

Keywords Warfarin, heparin, portal vein recanalization, bleeding

Ann Gastroenterol 2020; 33 (6): 1-7

\section{Introduction}

Liver cirrhosis is a clinical condition traditionally associated with bleeding risk (i.e., coagulopathy). This is due to multiple mechanisms, including prolonged bleeding time, increased

Department of anternal Medicine, University of Toledo, Toledo, $\mathrm{OH}$ (Sami Ghazaleh, Azizullah Beran, Kanana Aburayyan, Christian Nehme, Dipen Patel, Yasmin Khader, Sachit Sharma, Muhammad Aziz); ${ }^{\mathrm{b}}$ Gastroenterology and Hepatology, University of Tennessee, Memphis, TN (Yousef Abdel-Aziz); ' $\mathrm{G}$ astroenterology and Hepatology, Baylor College of Medicine, Houston, TX (Tariq Hammad);

${ }^{\mathrm{d}}$ Gastroenterology and Hepatology, University of Toledo, Toledo, $\mathrm{OH}$ (Ali Nawras), USA

Conflict of Interest: None

Correspondence to: Sami Ghazaleh, MD, University of Toledo, 2100 West Central Ave, Toledo, $\mathrm{OH}$ 43606, USA,

e-mail: sami.ghazaleh@utoledo.edu

Received 27 May 2020; accepted 31 July 2020;

published online 2 October 2020

DOI: https://doi.org/10.20524/aog.2020.0544 prothrombin time/international normalized ratio, decreased liver synthesis of clotting factors, thrombocytopenia, and hypofibrinolytic status [1]. In addition, liver cirrhosis is frequently complicated by esophageal varices, which further increases the risk of bleeding [2]. However, patients with liver cirrhosis are also at a high risk for venous thrombosis, especially portal vein (PV) thrombosis (PVT) [1]. Decreased levels of anticoagulant factors (protein $\mathrm{C}$, protein $\mathrm{S}$, and plasminogen), endothelial activation, disruption of the endothelial glycocalyx, and the generation of procoagulant microparticles all contribute to a prothrombotic status in cirrhotic patients [1]. Ultimately, the hemostatic balance in cirrhotic patients is fragile and may easily tend towards either a hypo- or a hypercoagulable status [1].

PVT is a frequent complication of liver cirrhosis, especially at the advanced stages [3]. Non-malignant PVT develops in $0.6-26 \%$ of patients with liver cirrhosis, depending on the severity of the cirrhosis, and the occurrence increases with cirrhosis progression [4]. Decreased PV velocity is determined to be the most important factor for development of PVT in patients with liver cirrhosis [5]. Anticoagulation has shown a proven benefit in the treatment of PVT in non-cirrhotic 
patients [6]. However, the role of anticoagulation in treating PVT in the setting of liver cirrhosis remains unclear because of the presumably greater risk of bleeding in cirrhotic patients [6].

We conducted a systematic review and meta-analysis of all published studies investigating the outcomes of anticoagulation in non-malignant PVT in cirrhotic patients compared with the outcomes of those who did not receive anticoagulation. The outcomes assessed were the efficacy of anticoagulation in achieving PV recanalization and the occurrence of variceal and any bleeding events.

\section{Materials and methods}

\section{Data sources and search strategy}

We performed a comprehensive search for published studies indexed in PubMed/MEDLINE, EMBASE, and the Cochrane Central Register of Controlled Trials from inception to August 2019. We also performed a manual search for additional relevant studies using references of the included articles. The following were the main search terms: ("portal vein thrombosis" or "portal venous thrombosis") and ("liver cirrhosis" or "hepatic cirrhosis") and ("anticoagulation" or "anticoagulant"). Supplementary Table 1 describes the full search term used in PubMed. We followed the Preferred Reporting Items for Systematic Reviews and Meta-Analyses (PRISMA) Statement guidelines to select the final studies [7]. Two investigators (SG and $\mathrm{AB}$ ) independently performed the search and shortlisted the studies for final review. Discrepancies were resolved by a third reviewer (KA). We assessed the quality of the included studies using the Newcastle-Ottawa Scale [8].

\section{Inclusion and exclusion criteria}

We included all available randomized controlled trials and cohort studies. Both full texts and abstracts were considered for the purpose of the manuscript. We excluded studies that included non-cirrhotic patients or malignant PVT. We also excluded single-arm studies, case reports, editorials, review articles, letters, guidelines and animal studies. No language limitation was applied.

\section{Data extraction}

The following data were extracted from the studies: study characteristics (author, publication year, study type, study design, and study population), type of anticoagulant used, number of patients in the anticoagulation group and control group, number of patients in whom PV recanalization was achieved (either partial or complete recanalization), and number of patients who developed bleeding (either variceal bleeding or any bleeding).

\section{Outcomes assessed}

The primary outcome assessed in our meta-analysis was the efficacy of anticoagulation in achieving PV recanalization. Our secondary outcomes were the occurrence of variceal bleeding and any bleeding events.

\section{Statistical analysis}

We performed a meta-analysis of the included studies using Review Manager 5.3 (Cochrane Collaboration, Copenhagen, The Nordic Cochrane Centre) and Comprehensive MetaAnalysis (Biostat, Englewood, USA). A random-effects model was applied, considering the presumed heterogeneity due to the patient population, type of anticoagulation and patient setting involved. Higgins $I^{2}$ test was used to assess for heterogeneity [9]. $I^{2}$ values greater than $50 \%$ implied the presence of substantial heterogeneity. A P-value of $<0.05$ was considered statistically significant. Weighted pooled relative risks (RRs) and 95\% confidence intervals (CIs) were calculated. Meta-regression was attempted to check if any variable had impacted our results.

\section{Bias assessment}

Bias assessment across studies was performed qualitatively, visualizing the funnel plot generated on review manager, as well as quantitatively, using Egger's regression analysis. A $\mathrm{P}$-value was generated using the Egger analysis and a value of $<0.05$ was associated with significant publication bias. If bias was present, further statistics using the Fail-Safe $\mathrm{N}$ test and Duval and Tweedie's "Trim and Fill" test were used to ascertain the impact of the bias.

\section{Results}

A total of 542 papers were retrieved by our search strategy. Among these, 33 were eligible for systemic review. Subsequently, we excluded 10 papers because of irrelevant population, 3 because of irrelevant interventions, and 11 because they had no control group. Eventually, 9 papers met our inclusion criteria and were included in the study [3-5,10-15]. Fig. 1 shows the PRISMA flow diagram that illustrates how the final studies were selected. Table 1 shows the baseline characteristics of the studies included in the meta-analysis. We did not contact the authors of the studies.

All 9 studies were published between 2011 and 2019 and included patients with non-malignant PVT. As regards their study design, all 9 studies included were observational; 6 studies were retrospective and 3 were prospective. Of the 9 included papers, 5 were full-text publications and 4 were abstracts. The total number of patients included in these studies was 474; 256 received anticoagulation for non-malignant PVT while 218 did not. The mean age of the treated group was similar to the untreated group ( 54.5 vs. 53.9 years). The proportion of male 
sex was $64.4 \%$ in the treated group compared to $48.8 \%$ in the untreated group. We assessed the quality of the included studies using the Newcastle-Ottawa Scale, shown in Supplementary Table 2. All studies scored $\geq 8$ on the scale.

\section{Primary outcome}

Table 2 summarizes the primary and secondary outcomes of the individual studies included in the meta-analysis. The

Table 1 Baseline characteristics of the studies included in the meta-analysis

\begin{tabular}{|c|c|c|c|c|c|c|c|c|}
\hline $\begin{array}{l}\text { Author, } \\
\text { year }\end{array}$ & $\begin{array}{l}\text { Publication } \\
\text { form }\end{array}$ & $\begin{array}{l}\text { Study } \\
\text { design }\end{array}$ & $\begin{array}{l}\text { Anticoagulated patients } \\
\text { and Controls (n) }\end{array}$ & $\begin{array}{l}\text { Age } \\
\text { (y) }\end{array}$ & Anticoagulant & $\begin{array}{l}\text { Anticoagulation } \\
\text { duration }(\mathrm{m})\end{array}$ & $\begin{array}{l}\text { Follow } \\
\text { up }(\mathrm{m})\end{array}$ & Indexes of LC severity \\
\hline $\begin{array}{l}\text { Chen, } \\
2015 \text { [3] }\end{array}$ & Full text & $\mathrm{R}$ & $\begin{array}{l}30 \text { treated } \\
36 \text { controls }\end{array}$ & $\begin{array}{l}44.9 \\
47.8\end{array}$ & Warfarin & 7.6 & 33 & $\begin{array}{l}\text { MELD: } 9.9 \\
\text { CTP: } 7.68 \\
\text { MELD: } 8.9 \\
\text { CTP: } 7.71\end{array}$ \\
\hline $\begin{array}{l}\text { Chung, } \\
2014[10]\end{array}$ & Full text & $\mathrm{R}$ & $\begin{array}{l}14 \text { treated } \\
14 \text { controls }\end{array}$ & $\begin{array}{l}59.4 \\
58.7\end{array}$ & Warfarin & 3.7 & 4 & $\begin{array}{l}\text { CTP: } A(6), B(8), C(0) \\
\text { CTP: } A(7), B(6), C(1)\end{array}$ \\
\hline $\begin{array}{l}\text { Copaci, } \\
2016[11]\end{array}$ & Abstract & $\mathrm{P}$ & $\begin{array}{l}50 \text { treated } \\
44 \text { controls }\end{array}$ & NR & Sulodexidum & 12 & 19 & MELD: 14.6 \\
\hline $\begin{array}{l}\text { Garcovich, } \\
2011[12]\end{array}$ & Abstract & $\mathrm{R}$ & $\begin{array}{l}15 \text { treated } \\
15 \text { controls }\end{array}$ & NR & LMWH & $3-6$ & 6 & CTP: A and B \\
\hline $\begin{array}{l}\text { Noronha } \\
\text { Ferreira, } \\
2019[4]\end{array}$ & Full text & $\mathrm{R}$ & $\begin{array}{l}37 \text { treated } \\
43 \text { controls }\end{array}$ & $\begin{array}{l}60 \\
59\end{array}$ & $\begin{array}{l}\text { Warfarin \& } \\
\text { LMWH }\end{array}$ & 10 & 25.5 & $\begin{array}{l}\text { MELD: } 14 \\
\text { CTP: A(12), B(16), C(9) } \\
\text { MELD: } 16 \\
\text { CTP: A(9), B(18), C(16) }\end{array}$ \\
\hline $\begin{array}{l}\text { Risso, } \\
2014[13]\end{array}$ & Abstract & $\mathrm{R}$ & $\begin{array}{l}50 \text { treated } \\
20 \text { controls }\end{array}$ & NR & NR & NR & NR & NR \\
\hline $\begin{array}{l}\text { Scheiner, } \\
2018 \text { [14] }\end{array}$ & Full text & $\mathrm{R}$ & $\begin{array}{l}12 \text { treated }^{*} \\
36 \text { controls }^{-}\end{array}$ & 52.9 & $\begin{array}{l}\text { LMWH or } \\
\text { phenprocoumon }\end{array}$ & 12 & 44.1 & $\begin{array}{l}\text { MELD: } 13.6 \\
\text { CTP: A(14), B(19), } \\
\text { C(18) }\end{array}$ \\
\hline $\begin{array}{l}\text { Senzolo, } \\
2012[5]\end{array}$ & Full text & $\mathrm{P}$ & $\begin{array}{l}35 \text { treated } \dagger \\
21 \text { controls }\end{array}$ & $\begin{array}{l}55.5 \\
52.3\end{array}$ & LMWH & 6 & 24 & $\begin{array}{l}\text { MELD: } 12.6 \\
\text { CTP: A(11), B(16), C(8) } \\
\text { MELD: } 13.7 \\
\text { CTP: A(5), B(9), C(7) }\end{array}$ \\
\hline $\begin{array}{l}\text { Tiwari, } \\
2018[15]\end{array}$ & Abstract & $\mathrm{P}$ & $\begin{array}{l}25 \text { treated } \\
20 \text { controls }\end{array}$ & 53.6 & $\begin{array}{l}\text { LMWH (9) or } \\
\text { heparin (16) }\end{array}$ & NR & 12 & $\begin{array}{l}\text { CTP: A(10), B(20), } \\
\text { C(15) }\end{array}$ \\
\hline
\end{tabular}

${ }^{\star}$ Initially, 51 patients were enrolled. 3 patients died early during follow up and were excluded from further analysis. 12 patients were treated with long-term anticoagulation ( $\geq 9$ months) and 36 patients received no anticoagulation or short-term anticoagulation

$\dagger$ Two patients were excluded after enrollment

CTP, Child-Turcotte-Pugh; LC, liver cirrhosis; LMWH, low molecular weight heparin; MELD, model for end-stage liver disease; $m$, month; NR, not reported; $P$, prospective; $R$, retrospective; $y$, year

Table 2 Primary and secondary outcomes of the individual studies included in the meta-analysis

\begin{tabular}{|c|c|c|c|c|c|c|}
\hline \multirow[t]{2}{*}{ Study } & \multicolumn{2}{|c|}{ PVT recanalization, $\mathrm{n}(\%)$} & \multicolumn{2}{|c|}{ Variceal bleeding, n (\%) } & \multicolumn{2}{|c|}{ Any bleeding, n (\%) } \\
\hline & Anticoagulation & $\begin{array}{c}\text { No } \\
\text { anticoagulation }\end{array}$ & Anticoagulation & $\begin{array}{c}\text { No } \\
\text { anticoagulation }\end{array}$ & Anticoagulation & $\begin{array}{c}\text { No } \\
\text { anticoagulation }\end{array}$ \\
\hline Chen, 2015 [3] & $15(68.2 \%)$ & $4(25.0 \%)$ & NR & NR & $8(36.4 \%)$ & $0(0.0 \%)$ \\
\hline Chung, 2014 [10] & $11(78.6 \%)$ & $5(35.7 \%)$ & $0(0.0 \%)$ & $1(7.1 \%)$ & $0(0.0 \%)$ & $2(14.3 \%)$ \\
\hline Copaci, 2016 [11] & $31(62.0 \%)$ & $12(27.3 \%)$ & NR & NR & NR & NR \\
\hline Garcovich, 2011 [12] & $7(46.7 \%)$ & $5(33.3 \%)$ & NR & NR & NR & NR \\
\hline Noronha Ferreira, 2019 [4] & $18(51.4 \%)$ & $6(18.8 \%)$ & $0(0.0 \%)$ & $4(12.5 \%)$ & $0(0.0 \%)$ & $4(12.5 \%)$ \\
\hline Risso, 2014 [13] & $37(74.0 \%)$ & $8(40.0 \%)$ & NR & NR & NR & NR \\
\hline Scheiner, 2018 [14] & $7(58.3 \%)$ & $10(27.8 \%)$ & $0(0.0 \%)$ & $9(25.0 \%)$ & $0(0.0 \%)$ & $16(44.4 \%)$ \\
\hline Senzolo, 2012 [5] & $21(63.6 \%)$ & $1(4.8 \%)$ & $1(3.0 \%)$ & $5(23.8 \%)$ & $4(12.1 \%)$ & $5(23.8 \%)$ \\
\hline Tiwari, 2018 [15] & $20(80.0 \%)$ & $4(20.0 \%)$ & NR & NR & NR & NR \\
\hline
\end{tabular}

$N R$, not reported; $P V T$, portal vein thrombosis 


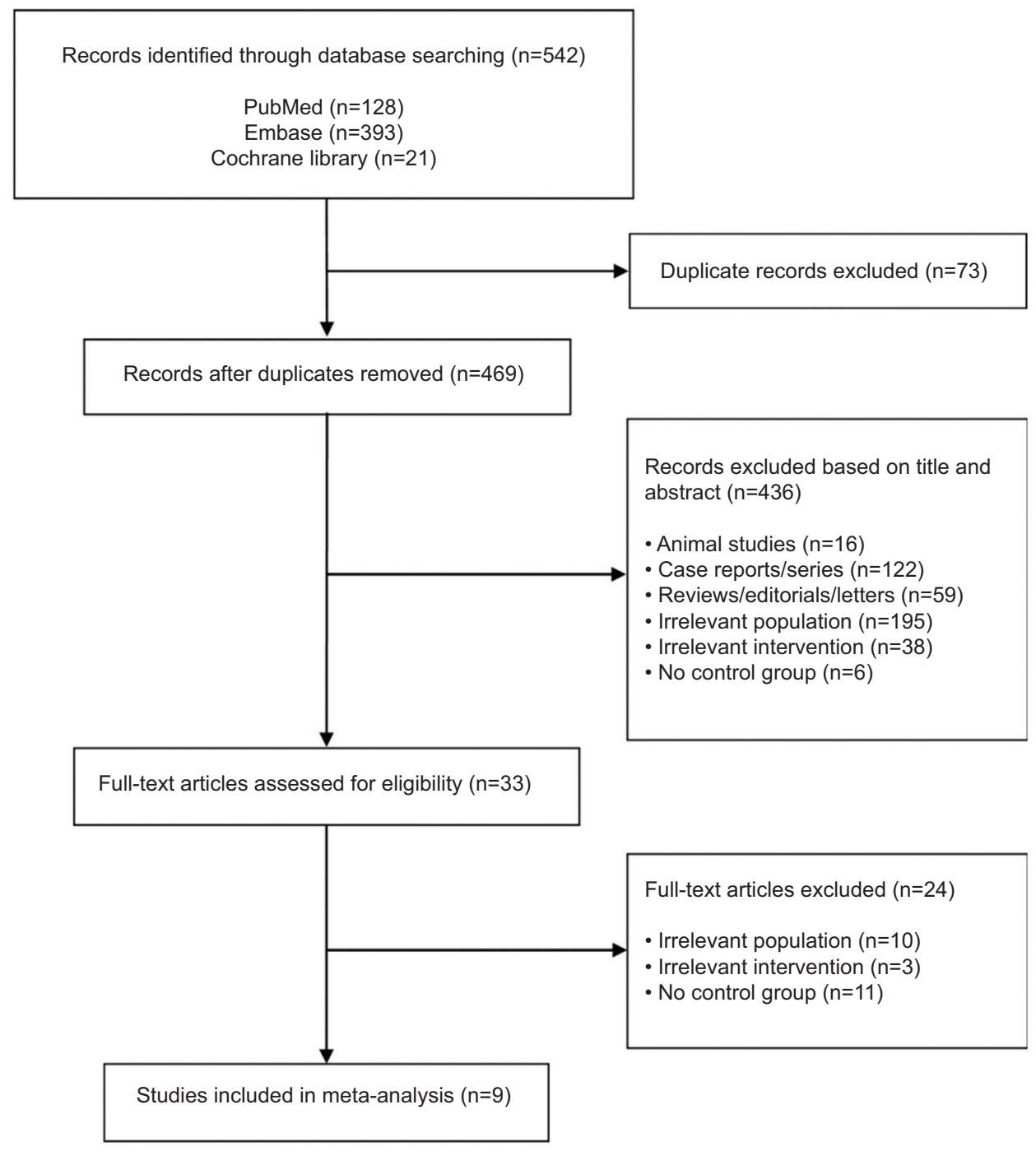

Figure 1 PRISMA flow diagram for selection of studies

rate of PV recanalization in patients with non-malignant PVT was significantly higher in the anticoagulated group compared to the untreated group $(65.2 \%$ vs. $25.2 \%$; RR 2.31, 95\%CI 1.80-2.96; $\mathrm{P}<0.00001 ; I^{2}=0 \%$ ) (Fig. 2). Metaregression of all studies evaluating PV recanalization did not reveal any significant moderating effect of publication year $(\mathrm{P}=0.46)$, study design $(\mathrm{P}=0.63)$ or publication type $(\mathrm{P}=0.76)$ (Supplementary Fig. 1, 2). Similarly, meta-regression of the studies that reported the severity and etiology of liver cirrhosis did not show the model for end-stage liver disease score $(\mathrm{P}=0.56)$ or etiology $(\mathrm{P}=0.88)$ as moderators of $\mathrm{PV}$ recanalization (Supplementary Fig. 3, 4).

\section{Secondary outcomes}

The risk of variceal bleeding was reported in 4 studies. These studies included a total of 197 patients, of whom 94 received anticoagulation and 103 did not. The risk of variceal bleeding was significantly lower in the anticoagulated group than in the untreated group $(0.1 \%$ vs. $18.5 \%$; RR $0.15,95 \% \mathrm{CI}$ $0.04-0.55 ; \mathrm{P}=0.004 ; I^{2}=0 \%$ ) (Fig. $3 \mathrm{~A}$ ). The risk of any bleeding was reported in 5 studies. These studies included 235 patients, of whom 116 received anticoagulation and 119 did not. The difference in the risk for any bleeding in both groups was not statistically significant ( $10.3 \%$ vs. $22.7 \%$; RR 0.43 , 95\%CI 0.09 1.99; $\mathrm{P}=0.28 ; I^{2}=52 \%$ ) (Fig. $3 \mathrm{~B}$ ).

\section{Subgroup analysis}

We conducted a subgroup analysis that included studies in which the anticoagulant used was low molecular weight heparin (LMWH). It showed that PV recanalization was significantly higher in the anticoagulated group compared to the control group $(64.2 \%$ vs $22.0 \%$; RR 2.76 , 95\%CI 1.41 5.41; $\mathrm{P}=0.003 ; I^{2}=54 \%$ ) (Fig. $4 \mathrm{~A}$ ). We conducted another 


\begin{tabular}{|c|c|c|c|c|c|c|c|c|c|}
\hline \multirow{2}{*}{ Study or Subgroup } & \multicolumn{2}{|c|}{ Anticoagulation } & \multicolumn{2}{|c|}{ No anticoagulation } & \multirow[b]{2}{*}{ Weight } & \multirow{2}{*}{$\begin{array}{c}\text { Risk Ratio } \\
\text { M-H, Random, } 95 \% \mathrm{Cl} \\
\end{array}$} & \multirow{2}{*}{\multicolumn{3}{|c|}{$\begin{array}{l}\text { Risk Ratio } \\
\text { M-H, Random, } 95 \% \mathrm{Cl}\end{array}$}} \\
\hline & Events & Total & Events & Total & & & & & \\
\hline Chen 2015 & 15 & 22 & 4 & 16 & $7.8 \%$ & $2.73[1.11,6.68]$ & & & \\
\hline Chung 2014 & 11 & 14 & 5 & 14 & $109 \%$ & $2.20[1.03,4.68]$ & & & \\
\hline Copaci 2016 & 31 & 50 & 12 & 44 & $22.2 \%$ & $2.27[1.34,3.86]$ & & $\longrightarrow$ & \\
\hline Garcovich 2011 & 7 & 15 & 5 & 15 & $7.7 \%$ & $1.40[0.57,3.43]$ & & & \\
\hline Noronha Ferreira 2019 & 18 & 35 & 6 & 32 & $10.0 \%$ & $2.74[1.25,6.04]$ & & $\longrightarrow$ & \\
\hline Risso 2014 & 37 & 50 & 8 & 20 & $19.7 \%$ & $1.85[1.06,3.24]$ & & $\longrightarrow$ & \\
\hline Scheiner 2018 & 7 & 12 & 10 & 36 & $12.3 \%$ & $2.10[1.03,4.28]$ & & $\rightarrow$ & \\
\hline Senzolo 2012 & 21 & 33 & 1 & 21 & $1.7 \%$ & $13.36[1.94,92.7]$ & & - & \\
\hline Tiwari 2018 & 20 & 25 & 4 & 20 & $7.7 \%$ & $4.00[1.63,9.82]$ & & & \\
\hline Total $(95 \% \mathrm{Cl})$ & & 256 & & 218 & $100.0 \%$ & $2.31[1.80,2.96]$ & & $V$ & \\
\hline \multirow{2}{*}{\multicolumn{8}{|c|}{$\begin{array}{l}\text { Total events } \\
\text { Heterogeneity: } \text { Tau }^{2}=0.00 ; \mathrm{Ch}^{2}=7.47, \mathrm{df}=8(\mathrm{P}=0.49) ; \mathrm{I}^{2}=0 \%\end{array}$}} & & \\
\hline & & & & & & & $\begin{array}{lcc}1 & 0.1 & 1 \\
\text { Favours no anticoagulation }\end{array}$ & $\begin{array}{c}10 \\
\text { Favours anticoagulation }\end{array}$ & 100 \\
\hline
\end{tabular}

Figure 2 Meta-analysis of studies that investigated the efficacy of anticoagulation in achieving portal vein recanalization in non-malignant portal vein thrombosis in patients with liver cirrhosis

CI, confidence interval

\begin{tabular}{|c|c|c|c|c|c|c|c|c|c|}
\hline \multirow{2}{*}{ A } & \multicolumn{2}{|c|}{ Anticoagulation } & \multirow{2}{*}{\multicolumn{3}{|c|}{ No anticoagulation }} & \multirow{2}{*}{\multicolumn{2}{|c|}{$\begin{array}{c}\text { Risk Ratio } \\
\text { M-H, Random, } 95 \% \mathrm{CI} \\
\end{array}$}} & \multirow{2}{*}{\multicolumn{2}{|c|}{$\begin{array}{c}\text { Risk Ratio } \\
\text { M-H, Random, 95\% CI }\end{array}$}} \\
\hline & Events & Total & & & & & & & \\
\hline Chung 2014 & 0 & 14 & 1 & 14 & $17.6 \%$ & $0.33[0.01,7.55]$ & & & \\
\hline Noronha Ferreira 2019 & 0 & 35 & 4 & 32 & $20.6 \%$ & $0.10[0.01,1.82]$ & & & \\
\hline Scheiner 2018 & 0 & 12 & 9 & 36 & $22.2 \%$ & $0.15[0.01,2.40]$ & & & \\
\hline Senzolo 2012 & 1 & 33 & 5 & 21 & $39.6 \%$ & $0.13[0.02,1.01]$ & & & \\
\hline Total $(95 \% \mathrm{Cl})$ & & 94 & & 103 & $100.0 \%$ & $0.15[0.04,0.55]$ & & & \\
\hline \multirow{2}{*}{\multicolumn{6}{|c|}{$\begin{array}{l}\text { Total events } \\
\text { Heterogeneity: } \text { Tau }^{2}=0.00 ; \mathrm{Ch}^{2}=0.35, \mathrm{df}=3(\mathrm{P}=0.95) ; \mathrm{I}^{2}=0 \% \\
\text { Test for overall effect: } Z=2.85(P=0.004)\end{array}$}} & & & & \\
\hline & & & & & & & 0.01 & $\begin{array}{cc}0.1 & 1 \\
\text { Favours anticoagulation }\end{array}$ & $\begin{array}{cc}10 & 100 \\
\text { Favours no anticoagulation }\end{array}$ \\
\hline \multicolumn{10}{|c|}{ A atio - } \\
\hline Study or Subgroup & $\begin{array}{l}\text { Anticoag } \\
\text { Events }\end{array}$ & $\begin{array}{l}\text { ulation } \\
\text { Total }\end{array}$ & $\begin{array}{l}\text { No anticoa } \\
\text { Events }\end{array}$ & $\begin{array}{l}\text { yulation } \\
\text { Total }\end{array}$ & Weight & $\begin{array}{c}\text { Risk Ratio } \\
\text { M-H, Random, 95\% }\end{array}$ & ${ }_{6} \mathrm{Cl}$ & \multicolumn{2}{|c|}{$\begin{array}{c}\text { Risk Ratio } \\
\text { M-H, Random. } 95 \% \mathrm{CI}\end{array}$} \\
\hline Chen 2015 & 8 & 22 & 0 & 16 & $17.3 \%$ & $12.57[0.78,203.03]$ & & & $\rightarrow$ \\
\hline Chung 2014 & 0 & 14 & 2 & 14 & $16.2 \%$ & $0.20[0.01,3,82]$ & & & \\
\hline Noronha Ferreira 2019 & 0 & 35 & 4 & 32 & $16.6 \%$ & $0.10[0.01,1.82]$ & 4 & & - \\
\hline Scheiner 2018 & 0 & 12 & 16 & 36 & $17.6 \%$ & $0.09[0.01,1.34]$ & & & - \\
\hline Senzolo 2012 & 4 & 33 & 5 & 21 & $32.2 \%$ & $0.51[0.15,1.68]$ & & & - \\
\hline Total $(95 \% \mathrm{Cl})$ & & 116 & & 119 & $100.0 \%$ & $0.43[0.09,1.99]$ & & & \\
\hline \multirow{2}{*}{\multicolumn{7}{|c|}{$\begin{array}{l}\text { lotal events } \\
\text { Heterogeneity: } \text { Tau }^{2}=1.54 ; \mathrm{Chi}^{2}=8.36, \mathrm{df}=4(P=0.08) ; l^{2}=52 \% \\
\text { Test for overall effect: } Z=1.08(P=0.28)\end{array}$}} & & & \\
\hline & & & & & & & 5.01 & $\begin{array}{c}1 \\
\text { Favours anticoagulation }\end{array}$ & $\begin{array}{c}10 \\
\text { Favours no anticoagulation }\end{array}$ \\
\hline
\end{tabular}

Figure 3 Meta-analysis of studies that investigated the safety of anticoagulation in non-malignant portal vein thrombosis in patients with liver cirrhosis. (a) Occurrence of variceal bleeding. (b) Occurrence of any bleeding

CI, confidence interval

subgroup analysis that included studies in which the anticoagulant used was warfarin. It also showed that PV recanalization was significantly higher in the anticoagulated group (72.2\% vs. $30.0 \%$; RR 2.41, 95\%CI 1.35-4.28; $\mathrm{P}=0.003$; $\left.I^{2}=0 \%\right)$ (Fig. 4B).

\section{Bias assessment}

There was visible gross asymmetry observed in the funnel blot, suggesting publication bias (Supplementary Fig. 5A). A significant trend towards positive reporting of any anticoagulation did appear to exist (Egger's test $\mathrm{P}=0.05$ ). The outcomes of our study did not change after implementing further statistics as mentioned previously (Supplementary Fig. 5B).

\section{Discussion}

This study was a systematic review and meta-analysis of published studies that assessed the effect of anticoagulation in patients with non-malignant PVT and liver cirrhosis, while excluding studies that included PVTs related to malignancy. We demonstrated that patients who received anticoagulation had a significantly higher rate of PV recanalization compared with those who did not receive anticoagulation $(65.2 \%$ vs. $25.2 \%$, respectively). Interestingly, our study showed that anticoagulation is associated with a lower incidence of variceal bleeding and any other bleeding events. This is consistent with a previous metaanalysis performed by Loffredo et al, which demonstrated that anticoagulant use is associated with a lower risk of variceal bleeding [16]. This finding can be explained by a decrease 


\begin{tabular}{|c|c|c|c|c|c|c|c|c|c|}
\hline \begin{tabular}{|l|} 
A \\
Study or Subgroup \\
\end{tabular} & \multicolumn{2}{|c|}{ Anticoagulation } & \multicolumn{3}{|c|}{ No anticoagulation } & $\begin{array}{c}\text { Risk Ratio } \\
\text { t } \mathrm{M}-\mathrm{H}, \text { Random, } 95 \% \mathrm{Cl} \\
\end{array}$ & \multicolumn{3}{|c|}{$\begin{array}{c}\text { Risk Ratio } \\
\text { M-H, Random, } 95 \% \mathrm{Cl}\end{array}$} \\
\hline Copaci 2016 & 31 & 50 & 12 & 44 & $37.7 \%$ & $227[1.34,386]$ & & \multirow{2}{*}{\multicolumn{2}{|c|}{$\square$}} \\
\hline Garcovich 2011 & 7 & 15 & 5 & 15 & $26.3 \%$ & \multirow{2}{*}{$\begin{array}{r}1.40[0.57,3.43] \\
13.36[1.94,92.07]\end{array}$} & & & \\
\hline Senzolo 2012 & 21 & 33 & 1 & 21 & $9.8 \%$ & & & $\longrightarrow$ & \\
\hline Tiwari 2018 & 20 & 25 & 4 & 20 & $26.2 \%$ & $4.00[1.63,9.82]$ & & $\longrightarrow$ & \\
\hline Total $(95 \% \mathrm{Cl})$ & \multicolumn{2}{|r|}{123} & & \multirow{2}{*}{\multicolumn{2}{|c|}{$100100.0 \%$}} & $2.76[1.41,5.41]$ & & & \\
\hline Total events & 79 & & 22 & & & & & & \\
\hline \multicolumn{6}{|c|}{$\begin{array}{l}\text { Heterogeneity: } \text { Tau }^{2}=0.24 ; \mathrm{Chi}^{2}=6.50, \mathrm{df}=3(P=0.09) ; \mathrm{I}^{2}=54 \% \\
\text { Test for overall effect: } Z=2.96(P=0.003)\end{array}$} & & $\begin{array}{lc}0.01 & 0.1 \\
& \text { Favours no anticoagulation } \\
\end{array}$ & $\begin{array}{c}10 \\
\text { Favours anticoagulation }\end{array}$ & \multirow[t]{3}{*}{100} \\
\hline \multirow{2}{*}{$\begin{array}{l}\text { B } \\
\text { Study or Subgroup }\end{array}$} & \multirow{2}{*}{\multicolumn{2}{|c|}{ Anticoagulation }} & \multicolumn{3}{|c|}{ No anticoagulation } & & \multirow{2}{*}{\multicolumn{2}{|c|}{$\begin{array}{c}\text { Risk Ratio } \\
\text { M-H, Random, } 95 \% \mathrm{Cl} \\
\end{array}$}} & \\
\hline & $\begin{array}{l}\text { Anticoag } \\
\text { Events }\end{array}$ & & Events & $\begin{array}{c}\text { Total } \\
\text { Total }\end{array}$ & Weight & $\begin{array}{c}\text { Risk Ratio } \\
\text { M-H, Random, } 95 \% \mathrm{CI}\end{array}$ & & & \\
\hline Chen 2015 & 15 & 22 & 4 & 16 & $41.5 \%$ & $2.73[1.11,6.68]$ & & 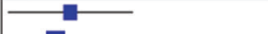 & \\
\hline Chung 2014 & 11 & 14 & 5 & 14 & $58.5 \%$ & $2.20[1.03,4.68]$ & & & \\
\hline Total $(95 \% \mathrm{Cl})$ & & 36 & & 30 & $100.0 \%$ & $2.41[1.35,4.28]$ & & & \\
\hline Total events & 26 & & 9 & & & & & & \\
\hline $\begin{array}{l}\text { Heterogeneity: Tau } 2= \\
\text { Test for overall effec }\end{array}$ & $\begin{array}{l}0.00 ; \mathrm{Chi}^{2} \\
\mathrm{t} Z=2,98\end{array}$ & $\begin{array}{l}=0.13, d \\
=0.00\end{array}$ & $\begin{array}{l}\text { If }=1(P=0.7 \\
\text { 3) }\end{array}$ & ); l= $0 \%$ & & & 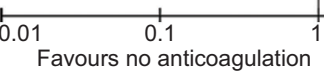 & $\begin{array}{c}10 \\
\text { Favours anticoagulation }\end{array}$ & $\overrightarrow{100}$ \\
\hline
\end{tabular}

Figure 4 Subgroup analysis of studies that investigated the efficacy of anticoagulation in achieving portal vein recanalization in non-malignant portal vein thrombosis in patients with liver cirrhosis, based on anticoagulant drug used. (a) Studies that used heparin or low molecular weight heparin. (b) Studies that used warfarin

CI, confidence interval

in portal and intrahepatic vascular resistance that ultimately prevents variceal rupture and bleeding $[14,16]$. However, we recommend endoscopic screening for varices and initiation of primary or secondary prophylactic measures of variceal bleeding before starting anticoagulation treatment in those patients [17].

PVT is a common sequela of liver cirrhosis and its occurrence increases with cirrhosis progression [3]. A recent study reported that patients with advanced liver cirrhosis have an annual PVT incidence of about 10-15\% [18]. Reduced PV velocity is considered the main contributor to the development of PVT in cirrhotic patients [5].

Anticoagulation provides a proven benefit in the treatment of PVT in non-cirrhotic patients [18]. However, the role of anticoagulation in treating PVT in the setting of liver cirrhosis remains unclear, because the data supporting starting anticoagulation are based mainly on retrospective small cohort studies and there is a lack of randomized controlled trials [18]. Although some studies have reported that spontaneous recanalization of PV can occur in up to $40 \%$ of cases, several studies have reported that the use of anticoagulation in cirrhotic patients achieves significant recanalization compared to those who did not receive anticoagulation $[19,20]$. A study by Francoz et al showed that no patients achieved recanalization in the group without anticoagulation, while $42 \%$ achieved recanalization in the anticoagulation group [20]. Therefore, based on our study findings, we recommend that cirrhotic patients with nonmalignant PVT should be treated with anticoagulation to avoid thrombus extension into the mesenteric veins, which can lead to complications including intestinal infarction [21].

Patients with liver cirrhosis can develop malignant PVT (caused by hepatocellular carcinoma) or non-malignant PVT (caused by venous stasis and portal hypertension) [22]. To date, only 2 meta-analyses have investigated the efficacy and safety of anticoagulation in the treatment of PVT in cirrhotic patients $[16,23]$. Both meta-analyses included malignant and non-malignant PVT. Given the fact that malignant and nonmalignant PVT are inherently different, we applied stricter exclusion criteria and excluded studies that included patients with malignant PVT. Further, newer studies have been reported, and we provide an updated meta-analysis based on recent publications. Our meta-analysis results are similar to those from a recent meta-analysis by Loffredo et al which showed that the rate of PV recanalization was significantly higher (71\% vs. $42 \%$ ) in patients receiving anticoagulation, without major bleeding events or increased variceal bleeding [16].

Subgroup analysis showed that treatment with heparin/LMWH (used in 223 patients) was effective in PV recanalization, with an RR of 2.76 (95\%CI 1.41-5.41; $\mathrm{P}=0.003)$. Similarly, warfarin (used in 66 patients) had similar effectiveness in PV recanalization, with an RR of 2.41 (95\%CI 1.35-4.28; $\mathrm{P}=0.003)$. The role of direct oral anticoagulants (DOACs) in patients with cirrhosis is still unknown, because most studies that evaluated their efficacy have excluded patients with liver disease [21]. However, Intagliata et al performed a first clinical study assessing the use of DOACs in patients with cirrhosis [24]. In that retrospective study, bleeding events did not differ significantly between patients who received DOACs compared with patients who received traditional anticoagulation (warfarin or LMWH) [21,24]. Therefore, it would be interesting to assess the impact of DOACs in cirrhotic patients with non-malignant PVT.

There are certain limitations to our meta-analysis. First, the studies included had a small sample size. Our meta-analysis only included 9 studies with a total of 474 patients. Second, because the literature lacks randomized controlled trials, our analysis only included observational studies. Future research should involve randomized controlled trials that report high quality evidence with large sample sizes to confirm our results. Third, only one study reported the subjects' survival rates. Long follow-up studies are needed to assess the long-term efficacy and the potential mortality benefit of anticoagulation use. Fourth, we could not assess the quality of the abstracts included in the analysis, which might affect the validity of our conclusion. 
Despite the limitations, our updated meta-analysis recruited studies using stringent inclusion and exclusion criteria. The included observational studies were of high quality based on Newcastle-Ottowa scoring. Our results were consistent across all subgroup analyses and are in line with previous reported meta-analyses. We further attempted a meta-regression analysis based on publication year, publication type, study design, and severity and etiology of cirrhosis, which did not significantly alter our primary outcome.

In conclusion, our study supports anticoagulation use in patients with non-malignant PVT and cirrhosis, since it is associated with a high rate of PV recanalization without any obvious increase in bleeding risk. Despite the inspiring outcome in our meta-analysis, we emphasize that all the existing evidence is of low quality in the absence of randomized controlled trials. Accordingly, it is imperative to perform a randomized controlled study to validate the efficacy and safety of anticoagulation in the treatment of non-malignant PVT in the setting of liver cirrhosis. Future studies should also investigate the optimal duration of anticoagulation. Ultimately, treatment of PVT with anticoagulation should be considered on a case-by-case basis to maximize patient benefits and limit the risk of bleeding.

\section{Summary Box}

\section{What is already known:}

- Anticoagulation is the mainstay treatment for portal vein thrombosis (PVT) in non-cirrhotic patients

- Liver cirrhosis is associated with hypercoagulability and coagulopathy

- Previous meta-analyses have investigated the role of anticoagulation for PVT in cirrhotic patients, but have not made the distinction between malignant and non-malignant PVT

\section{What the new findings are:}

- Anticoagulation is effective in achieving portal vein recanalization in non-malignant PVT in cirrhotic patients

- In this population, anticoagulation does not increase the risk of any bleeding, and may even lower the risk of variceal bleeding

- Portal vein recanalization in this population can be achieved regardless of whether low molecular weight heparin or warfarin is used

\section{References}

1. Lisman T, Porte RJ. Pathogenesis, prevention, and management of bleeding and thrombosis in patients with liver diseases. Res Pract Thromb Haemost 2017;1:150-161.

2. de Franchis R. Expanding consensus in portal hypertension: Report of the Baveno VI Consensus Workshop: Stratifying risk and individualizing care for portal hypertension. J Hepatol 2015;63:743-752.

3. Chen H, Liu L, Qi X, et al. Efficacy and safety of anticoagulation in more advanced portal vein thrombosis in patients with liver cirrhosis. Eur J Gastroenterol Hepatol 2016;28:82-89.

4. Noronha Ferreira C, Reis D, Cortez-Pinto H, et al. Anticoagulation in cirrhosis and portal vein thrombosis is safe and improves prognosis in advanced cirrhosis. Dig Dis Sci 2019;64:2671-2683.

5. Senzolo M, Sartori TM, Rossetto V, et al. Prospective evaluation of anticoagulation and transjugular intrahepatic portosystemic shunt for the management of portal vein thrombosis in cirrhosis. Liver Int 2012;32:919-927.

6. DeLeve LD, Valla D-C, Garcia-Tsao GA; American Association for the Study of Liver Diseases. Vascular disorders of the liver. Hepatology (Baltimore, Md) 2009;49:1729-1764.

7. Moher D, Liberati A, Tetzlaff J, Altman DG; PRISMA Group. Preferred reporting items for systematic reviews and metaanalyses: the PRISMA statement. PLoS Med 2009;6:e1000097.

8. Deeks JJ, Dinnes J, D’Amico R, et al. Evaluating non-randomised intervention studies. Health Technol Assess 2003;7:iii-x.

9. Higgins JP, Thompson SG, Deeks JJ, Altman DG. Measuring inconsistency in meta-analyses. BMJ 2003;327:557-560.

10. Chung JW, Kim GH, Lee JH, et al. Safety, efficacy, and response predictors of anticoagulation for the treatment of nonmalignant portal-vein thrombosis in patients with cirrhosis: a propensity score matching analysis. Clin Mol Hepatol 2014;20:384-391.

11. Copaci I, Micu L, Ioanitescu S, Chiriac G, Lupescu I. UEG Week 2016 Poster Presentations. United European Gastroenterol J 2016;4:A157-A720.

12. Garcovich M, Zocco MA, Ainora ME, et al. Clinical outcome of portal vein thrombosis (PVT) in cirrhotic patients: observe or treat? Hepatology 2011;54:360A-1455A.

13. Risso A, Stradella D, Martini S, Rizzetto M, Salizzoni M. Liver transplantation in cirrhotic patients with portal vein thrombosis: A single centre experience. Dig Liver Dis 2014;46:e40.

14. Scheiner B, Stammet PR, Pokorny S, et al. Anticoagulation in nonmalignant portal vein thrombosis is safe and improves hepatic function. Wien Klin Wochenschr 2018;130:446-455.

15. Tiwari LP, Venkateswaran AR, Solomon TR, Mohamed KS, Kini R, Tiwari LP. Assessment of effect of anticoagulation for portal vein recanalization in non-tumoral portal vein thrombosis with cirrhosis. J Clin Exper Hepatol 2018;8:S75-S76.

16. Loffredo L, Pastori D, Farcomeni A, Violi F. Effects of anticoagulants in patients with cirrhosis and portal vein thrombosis: a systematic review and meta-analysis. Gastroenterology 2017;153:480-487.

17. Chen H, Turon F, Hernandez-Gea V, et al. Nontumoral portal vein thrombosis in patients awaiting liver transplantation. Liver Transpl 2016;22:352-365.

18. Intagliata NM, Caldwell SH, Tripodi A. Diagnosis, development, and treatment of portal vein thrombosis in patients with and without cirrhosis. Gastroenterology 2019;156:1582-1599.

19. Raja K, Jacob M, Asthana S. Portal vein thrombosis in cirrhosis. J Clin Experiment Hepatol 2014;4:320-331.

20. Francoz C, Belghiti J, Vilgrain V, et al. Splanchnic vein thrombosis in candidates for liver transplantation: usefulness of screening and anticoagulation. Gut 2005;54:691-697.

21. Faccia M, Ainora ME, Ponziani FR, et al. Portal vein thrombosis in cirrhosis: Why a well-known complication is still matter of debate. World J Gastroenterol, 2019;25:4437-4451.

22. Tublin ME, Dodd GD $3^{\text {rd }}$, Baron RL. Benign and malignant portal vein thrombosis: differentiation by CT characteristics. AJR Am J Roentgenol 1997;168:719-723.

23. Qi X, De Stefano V, Li H, Dai J, Guo X, Fan D. Anticoagulation for the treatment of portal vein thrombosis in liver cirrhosis: a systematic review and meta-analysis of observational studies. Eur J Intern Med 2015;26:23-29.

24. Intagliata NM, Henry $\mathrm{ZH}$, Maitland $\mathrm{H}$, et al. Direct oral anticoagulants in cirrhosis patients pose similar risks of bleeding when compared to traditional anticoagulation. Dig Dis Sci 2016;61:1721-1727. 


\section{Supplementary material}

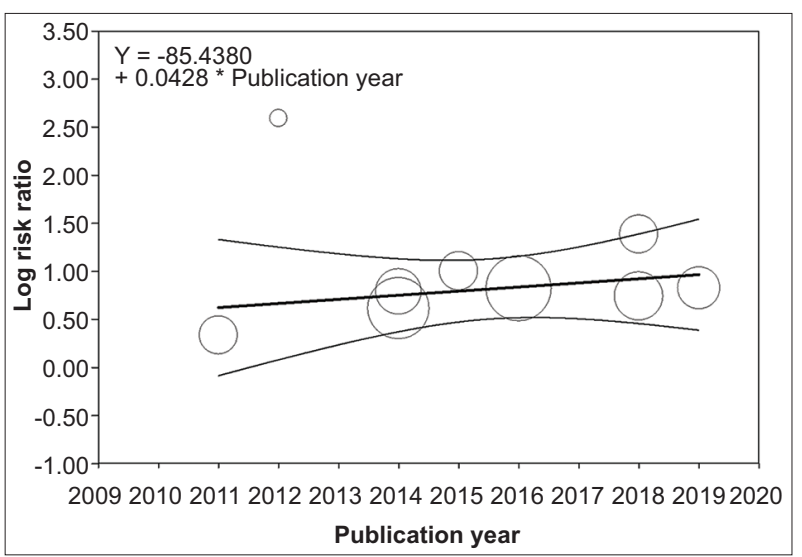

Supplementary Figure 1 Regression of log risk ratio on publication year. Meta-regression model did not demonstrate significant moderating effect of year of publication $(\mathrm{P}=0.46)$. (Note: bubbles represent study, size of the bubble represents study weight, central thick line represents meta-regression line, curved upper and lower lines represent $95 \%$ confidence interval)

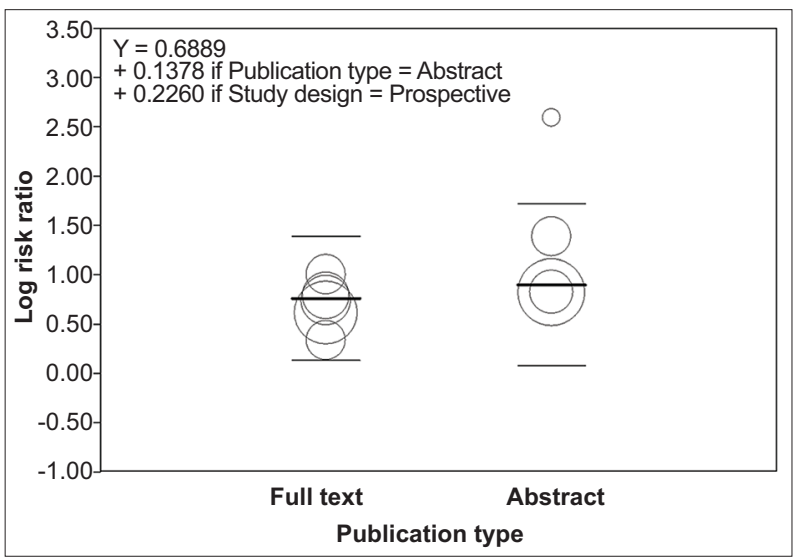

Supplementary Figure 2 Regression of log risk ratio on publication type. Meta-regression model did not demonstrate significant moderating effect based on study design $(\mathrm{P}=0.63)$ and publication type $(\mathrm{P}=0.76)$. (Note: bubbles represent a study, size of the bubble represents study weight, central thick line represents meta-regression line, upper and lower thin lines represent 95\% confidence interval)

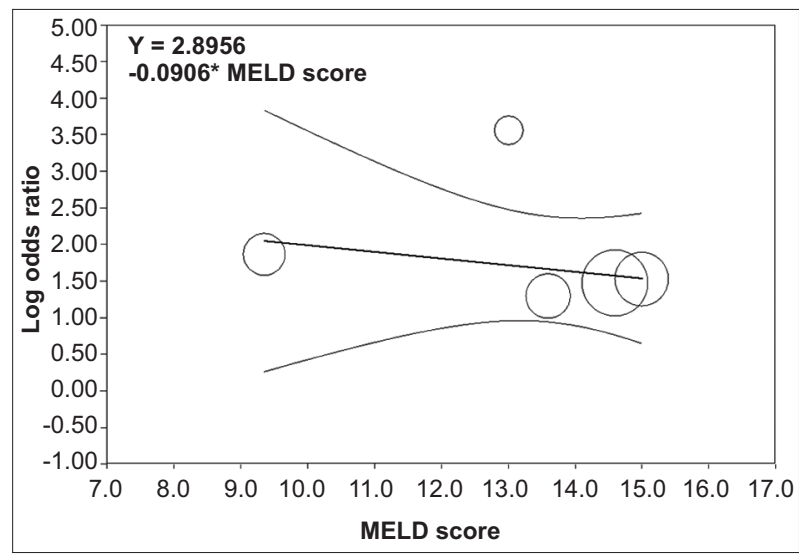

Supplementary Figure 3 Regression of log odds ratio on model for end-stage liver disease (MELD) score. Meta-regression model did not demonstrate significant moderating effect based on MELD score $(\mathrm{P}=0.56)$. (Note: bubbles represent study, size of the bubble reprsents study weight, central thick line represents meta-regression line, curved upper and lower lines represent $95 \%$ confidence interval)

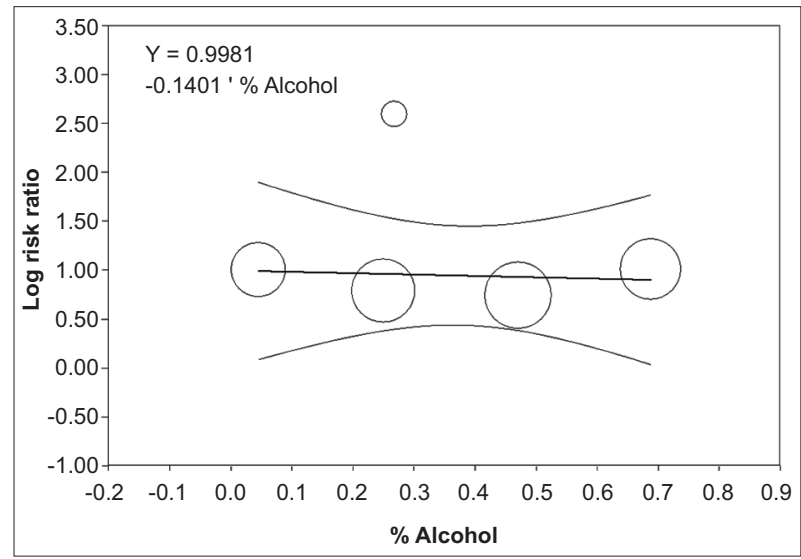

Supplementary Figure 4 Regression of log risk ratio on the percentage of alcoholic cirrhosis as the etiology of liver cirrhosis. Meta-regression model did not demonstrate significant moderating effect based on the percentage of alcoholic cirrhosis as the etiology of liver cirrhosis $(\mathrm{P}=0.88)$. (Note: bubbles represent study, size of the bubble represents study weight, central thick line represents meta-regression line, curved upper and lower lines represent 95\% confidence interval) 


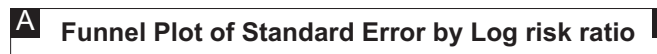

B Funnel Plot of Standard Error by Log risk ratio
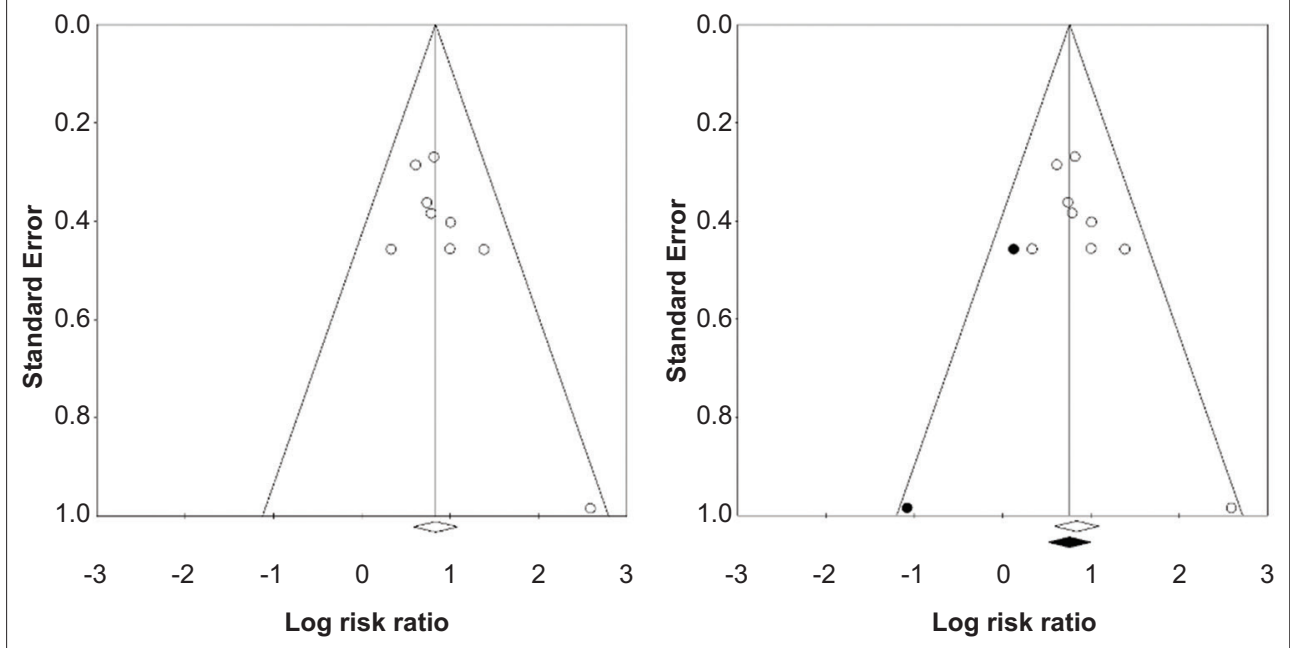

Supplementary Figure 5 Publication bias assessment. (A) Funnel plot demonstrating visible asymmetry suggesting publication bias after plotting actual studies using portal vein recanalization as outcome. (B) Adjusted funnel plot using the "trim and fill test" without significantly altering the outcomes (Note: Filled circles represent "additionally filled" studies)

Supplementary Table 1 Search strategy used in PubMed

Search Query

Items found

\begin{tabular}{|c|c|c|}
\hline$\# 1$ & 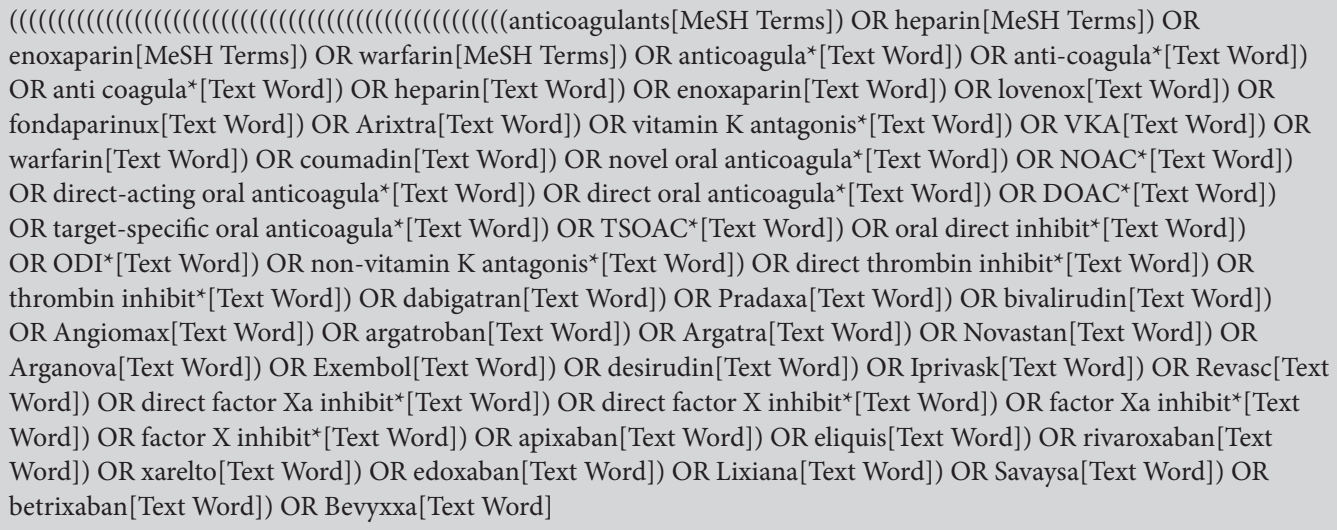 & 209254 \\
\hline$\# 2$ & $\begin{array}{l}(((((((\text { venous thrombosis[MeSH Terms]) OR blood coagulation[MeSH Terms]]) OR portal vein[MeSH Terms]) OR } \\
\text { thrombosis[MeSH Terms]) OR portal vein thrombosis[Text Word]) OR portal venous thrombosis[Text Word]) OR } \\
\text { PVT[Text Word]) OR (portal[Text Word] AND vein[Text Word] AND thrombosis[Text Word])) OR (portal[Text } \\
\text { Word] AND venous[Text Word] AND thrombosis[Text Word]) }\end{array}$ & 196402 \\
\hline$\# 3$ & $\begin{array}{l}((((((((((\text { liver cirrhosis[MeSH Terms]]) OR fibrosis[MeSH Terms]) OR liver cirrhosis[Text Word]) OR hepatic } \\
\text { cirrhosis[Text Word]) OR cirrhosis[Text Word]) OR liver fibrosis[Text Word]) OR hepatic fibrosis[Text Word]) OR } \\
\text { fibrosis[Text Word]) OR (liver[Text Word] AND cirrhosis[Text Word])) OR (hepatic[Text Word] AND cirrhosis[Text } \\
\text { Word])) OR (liver[Text Word] AND fibrosis[Text Word])) OR (hepatic[Text Word] AND fibrosis[Text Word]) }\end{array}$ & 330378 \\
\hline$\# 4$ & \#1 AND \#2 AND \#3 & 693 \\
\hline$\# 5$ & $\begin{array}{l}\text { ((((benign[Text Word]) OR nonmalignant[Text Word] }) \text { OR non malignant[Text Word]) OR stasis[Text Word]) OR } \\
\text { portal hypertension[Text Word] }\end{array}$ & 260606 \\
\hline$\# 6$ & \#4 AND \#5 & 147 \\
\hline$\# 7$ & $\begin{array}{l}\text { \#6 NOT (“case reports"[Publication Type] OR “editorial”[Publication Type] OR "guideline"[Publication Type] OR } \\
\text { "introductory journal article"[Publication Type] OR “meta analysis"[Publication Type] OR “news"[Publication Type] } \\
\text { OR "practice guideline"[Publication Type] OR "review"[Publication Type] OR "systematic review"[Publication Type]) }\end{array}$ & 128 \\
\hline
\end{tabular}


Supplementary Table 2 Quality assessment of the studies using the Newcastle-Ottawa Scale

\begin{tabular}{|c|c|c|c|c|c|c|}
\hline Criteria & Condition & $\begin{array}{l}\text { Chen } \\
2015\end{array}$ & $\begin{array}{l}\text { Chung } \\
2014\end{array}$ & $\begin{array}{l}\text { Noronha } \\
\text { Ferreira } 2019\end{array}$ & $\begin{array}{l}\text { Scheiner } \\
2018\end{array}$ & $\begin{array}{l}\text { Senzolo } \\
2012\end{array}$ \\
\hline \multicolumn{7}{|l|}{ Selection } \\
\hline Representativeness of exposed cohort & $\begin{array}{l}\text { Patients diagnosed with non-malignant } \\
\text { portal vein thrombosis who underwent } \\
\text { anticoagulation }\end{array}$ & * & * & * & * & * \\
\hline Selection of non-exposed cohort & $\begin{array}{l}\text { Patients diagnosed with non-malignant } \\
\text { portal vein thrombosis who did undergo } \\
\text { anticoagulation }\end{array}$ & * & * & * & * & * \\
\hline Ascertainment of exposure? & Secure records & * & * & * & * & * \\
\hline $\begin{array}{l}\text { Demonstration that outcome of interest } \\
\text { was not present at start of study? }\end{array}$ & Yes & * & * & * & * & * \\
\hline \multicolumn{7}{|l|}{ Comparability } \\
\hline Study controls for baseline imbalances? & Age and severity of liver cirrhosis & ** & ** & ** & * & ** \\
\hline \multicolumn{7}{|l|}{ Outcome } \\
\hline Assessment of outcome & Confirmation by ultrasound & * & * & * & * & * \\
\hline $\begin{array}{l}\text { Was follow up long enough for } \\
\text { outcomes to occur }\end{array}$ & 6 months & * & - & * & * & * \\
\hline Adequacy of follow up of cohorts & $\begin{array}{l}\text { Complete follow up or less than } 10 \% \text { lost to } \\
\text { follow up }\end{array}$ & * & - & * & * & * \\
\hline
\end{tabular}

A study can be awarded a star $\left.{ }^{*}\right)$ for a criterion if it satisfies the condition for that criterion. A study can be awarded 2 stars $\left({ }^{* *}\right)$ in the comparability item if it controls for both age and severity of liver cirrhosis 